Research Article

\title{
A Simple and Rapid Method for Quantitative HPLC MS/MS Determination of Selected Perfluorocarboxylic Acids and Perfluorosulfonates in Human Serum
}

\author{
Valentina Marra $(\mathbb{D}$, Annalisa Abballe $\mathbb{D}$, Elena Dellatte $(\mathbb{D}$, Nicola Iacovella $(\mathbb{D}$, \\ Anna Maria Ingelido $\mathbb{\infty}$, and Elena De Felip \\ Istituto Superiore di Sanità, Dipartimento Ambiente e Salute, Viale Regina Elena 299, Roma 00161, Italy \\ Correspondence should be addressed to Valentina Marra; valentina.marra@iss.it
}

Received 31 March 2020; Revised 9 September 2020; Accepted 3 October 2020; Published 16 October 2020

Academic Editor: David M. Lubman

Copyright (๑) 2020 Valentina Marra et al. This is an open access article distributed under the Creative Commons Attribution License, which permits unrestricted use, distribution, and reproduction in any medium, provided the original work is properly cited.

\begin{abstract}
Per- and polyfluorinated alkyl substances (PFASs) are ubiquitous environmental contaminants, present in the environment and in the human body. They have raised global concern because of their diffusion in the environment, particularly in water, causing cases of human overexposure due to consumption of contaminated drinking water. Human biomonitoring is the most effective way to characterize human exposure to PFASs, and it is important that as many labs as possible could easily perform this kind of analysis. Analytical methods for quantitation of PFAS mixtures in human serum have been developed, but most of them required materials that are not always easily available in all the laboratories. This paper describes a very simple and accessible HPLC MS/MS method of analysis and quantification of 13 perfluorocarboxylic acids and perfluorosulfonate compounds (belonging to the class of per- and polyfluorinated alkyl substances (PFASs)) in human serum. Method development data provide detailed descriptions of the optimization process in terms of sample preparation, laboratory analysis of human serum samples, determination of analytes by HPLC MS/MS, and describing the pump gradient time, working conditions, and acquisition.
\end{abstract}

\section{Introduction}

Perfluoroalkyl and polyfluoroalkyl substances (PFASs) are a class of synthetic organic chemicals containing fully (per) or partly (poly) fluorinated carbon chains with different functional groups used since 1950s in various industrial and commercial products (fire foams, clothes, protective coatings for carpets, pots, paints, and insecticides) [1]. For many years, they have spread in the environment (water, soil, and biota) and have also entered the food chain [2-6]. Their chemical-physical properties are thermal and chemical resistance, impermeability to oil and water, and surfactant properties. These chemical-physical properties combined with high industrial production and frequent and widespread use have made PFAS persistent contaminants capable of spreading easily in the environment without degrading but bioaccumulating and bioconcentrating in the food chain $[7,8]$. Human exposure to these substances can pose a risk to public health [9-13]; for this reason, the scientific community in the last few years is implementing a series of monitoring measures for these substances in the environment and in humans. In particular, human biomonitoring is the most effective way to characterize human exposure to PFAS. We therefore developed a very simple method for the analysis of PFAS in human serum by liquid chromatography tandem mass spectrometry. The sample preparation is based 
on the denaturation of proteins with acetonitrile, volume reduction, and injection: no extraction or purification in the solid phase is necessary.

\section{Materials and Methods}

The Laboratory of the Italian National Institute of Health (ISS), Human Exposure to Environmental Contaminants Unit, has developed the method described in this paper.

We ordinarily use the method hereafter described for the analysis of serum samples within the framework of various national and international projects and in interlaboratory comparison exercises [10, 14-18], Ring Exercise Test for Persistent Organic Pollutants in Human Serum https:// www.inspq.qc.ca/en/ctq/eqas/amap/description [19]. Results obtained by our laboratory in the interlaboratory comparison exercises are reported in detail in supplementary data 2. Reports of the interlaboratory comparison exercises are in supplementary data 3.

2.1. Sample Preparation. Sampling is usually performed by Italian local sanitary units in charge for blood withdrawal, which we provide with specific instructions to avoid PFAS contamination, particularly contaminations deriving from the presence of teflon-coated materials or similar (these are among the major causes of analytical interferences found in PFAS analysis). We recommend using polycarbonate or high-density polyethylene materials to collect or store the samples. Blood withdrawal (about $5 \mathrm{~mL}$ ) must be performed with a serum tube containing no anticoagulant (may contain coagulation activator and separator gel) that must be placed in a suitable rack in a vertical position (avoiding as far as possible any solicitation of the contents) and left at room temperature until the clot is completely formed (about $30 \mathrm{~min}$ ) and then centrifuged (centrifuge within 2 hours after collection) at about $3500 \mathrm{~g}$ for 15 minutes at $20^{\circ} \mathrm{C}$.

After centrifugation, the tube must be removed from the centrifuge and placed in a rack, and the serum was removed from the tube with a pipette, transferred into a $15 \mathrm{~mL}$ polypropylene tube with a properly labelled Falcon-type screw cap, and immediately frozen keeping the tube in a vertical position inside a suitable test tube rack. $15 \mathrm{~mL}$ polypropylene test tubes used for storing and shipping the serum samples must be identified by indicating the identification code of the sample on the label. The tubes containing the serum samples should be stored at $-20^{\circ} \mathrm{C}$ in a rack until shipment. Before analysis, the serum samples are allowed to warm up at room temperature and thoroughly mixed, and $250 \mu \mathrm{L}$ must be aspirated with a pipette, transferred to a $15 \mathrm{~mL}$ polypropylene centrifuge tube, weighed and spiked with $50 \mu \mathrm{L}$ of internal standard solution in acetonitrile (concentration: $210.40 \mathrm{ng} / \mathrm{mL}$ each PFAS), and allowed to rest overnight at $4^{\circ} \mathrm{C}$. The following steps are performed after samples have been allowed to equilibrate to room temperature for one hour. The spiked samples are mixed with acetonitrile and centrifuged (Megafuge $1.0 \mathrm{R}$ Heraeus Sepatech) at $3500 \mathrm{rpm}$ for $15 \mathrm{~min}$ to precipitate proteins. After centrifugation and separation of the two phases, the volume of the supernatant is reduced in a multiple sample evaporator system (Zymark TurboVap LV concentration evaporator) to about $200 \mu \mathrm{L}$, transferred to an autosampler vial, evaporated to dryness, and added with $300 \mu \mathrm{L}$ of the injection standard solution (13C4 PFHpA $50 \mathrm{ng} / \mathrm{mL}$ in acetonitrile) to undergo instrumental analysis. Extracts are stored at $4^{\circ} \mathrm{C}$ and analyzed within 1 week after conditioning at room temperature for one hour. Figure 1 shows in graphic form how human exposure to PFAS usually occurs (indicating the major sources of exposure) and how these compounds are analyzed in human body through blood sampling and analysis.

2.2. Chemicals. High-purity chemical ${ }^{13} \mathrm{C}$-labelled internal standards of perfluorobutanoic acid (PFBA), perfluorohexanoic acid (PFHxA), perfluorooctanoic acid (PFOA), perfluorohexane sulfonate (PFHxS), perfluorooctane sulfonate (PFOS), perfluorononanoic acid (PFNA), perfluorodecanoic acid (PFDA), perfluoroundecanoic acid (PFUdA), and perfluorododecanoic acid (PFDOA) are purchased from Wellington Laboratories (Wellington Laboratories Inc., Ontario, Canada, N1G 3M5). The ${ }^{13} \mathrm{C}$-labelled internal standard solution is prepared by withdrawing, with a $500 \mu \mathrm{L}$ syringe, $2.5 \mathrm{~mL}$ of a commercial mixture of PFAS $13^{\circ} \mathrm{C}$ or $18 \mathrm{O}$-labelled identified as "MPFAC-MXA," at a concentration of $2000 \mathrm{ng} / \mathrm{mL}$ diluted with acetonitrile up to the volume of $25 \mathrm{~mL}$ in a class A glass flask and stored in the refrigerator at $4 \pm 3^{\circ} \mathrm{C}$ until use. The serum samples are analyzed for nine perfluorocarboxylic acids: PFBA, PFPeA, PFHxA, PFHpA, PFOA, PFNA, PFDA, PFUdA, and PFDoA and four perfluorosulfonates: PFBS, PFHxS, PFHpS, and PFOS; unlabelled standards of these PFASs are purchased from Wellington Laboratories (Wellington Laboratories Inc., Ontario, Canada, N1G 3M5). The unlabelled PFAS standard solution identified as "PFAS," at a concentration of $200 \mathrm{ng} / \mathrm{mL}$, is prepared by withdrawing $1 \mathrm{~mL}$ of a commercial mixture of natural PFAS identified as "PFAC-MXB," at a concentration of $2000 \mathrm{ng} / \mathrm{mL}$ diluted with acetonitrile up to the volume of $10 \mathrm{~mL}$ in a glass class A flask. This solution is stored at $4 \pm 3^{\circ} \mathrm{C}$ until use. The injection standard solution $\left({ }^{13} \mathrm{C}_{4}\right.$ PFHpA $50 \mathrm{ng} / \mathrm{mL}$ in acetonitrile) identified as "13C4PFHpA" is prepared by withdrawing $1 \mathrm{~mL}$ of a commercial mixture of ${ }^{13} \mathrm{C}_{4}$ PFHpA $50 \mu \mathrm{g} / \mathrm{mL}$ and diluted with acetonitrile at a volume of $1000 \mathrm{~mL}$ in a glass class $\mathrm{A}$ flask. It is stored at $4 \pm 3^{\circ} \mathrm{C}$ until use. HPLC-grade acetonitrile ( $\geq 99.9 \%$ purity) is purchased from VWR Chemicals (Radnor, Pennsylvania, United States), and HPLC-grade water is purchased from J.T. Becker (Thermo Fisher Scientific, Hampton, New Hampshire, United States).

2.3. Instrumental Analysis. Instrumental analysis is carried out by HPLC (Waters Alliance 2695, Waters Corporation, Milford, MA, USA). Chromatographic separation is achieved using a Kinetex C18 Column $(5 \mu \mathrm{m}$, $100 \mathrm{~mm} \times 2.1 \mathrm{~mm}$ ID, $100 \AA$ ) supplied by Phenomenex (Torrance, CA, USA) operated at a temperature of $45^{\circ} \mathrm{C}$. A cartridge column (YMC ODS-A $5 \mu \mathrm{m}, 20 \times 4.0 \mathrm{~mm}$ ID, $120 \AA$ ) supplied by YMC Europe (Dinslaken, Germany) is attached in line before the column to trap residues from the mobile phase or the system. An injection volume of $10 \mu \mathrm{L}$ is 


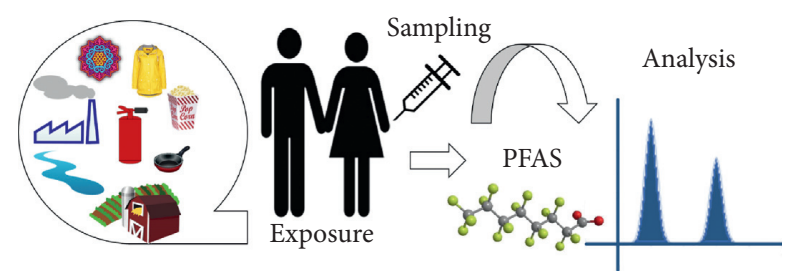

FIgUre 1: Assessment of human exposure to PFAS through biomonitoring.

determined to be optimal considering the required sensitivity of the method and the chromatographic performance. Larger injection volumes have been shown to cause matrix effect. The mobile phases are an acetic acid/ammonium acetate solution in water (A) and acetonitrile (B). Solution A is prepared by adding $19 \mathrm{mg}$ ammonium acetate and $4.5 \mathrm{~mL}$ acetic acid to $250 \mathrm{~mL}$ of water. We have investigated the effect of the $\mathrm{pH}$ of solution $\mathrm{A}$ on the chromatographic behavior of the analytes and found that an improvement of short-chained PFAS peak shapes is achieved at lower $\mathrm{pH}$ values; $\mathrm{pH} 2.24$ resulted to be good enough to analyze shortchained PFASs and acceptable to limit system deterioration. The flow during the injection is $0.35 \mathrm{~mL} / \mathrm{min}$, and the HPLC pump gradient timetable is shown in Table 1 . The HPLC is interfaced with a triple quadrupole mass spectrometer (Micromass Quattro micro TM API, Waters Corporation, Milford, MA, USA).

Analytes are detected by electrospray negative ionization in ion multiple reaction monitoring (MRM) mode, and argon is used as collision gas. A minimum of ten scans across the chromatographic peak is required to ensure adequate peak shape. Working conditions and acquisition parameters are reported in Tables 2 and 3.

\section{Results}

In human serum, perfluorosulfonates (particularly, PFHxS and PFOS) consist of significant quantities of branched isomers, whereas perfluorocarboxylic acids are predominantly linear. In our method, we quantify perfluorosulfonates as the sum of linear and branched isomers. For PFOS quantification, we selected the transition $499>80$ as it is more sensitive than the $\mathrm{m} / z 499>99$ transition and generally preferred when quantifying both linear and branched isomers. Data acquisition and processing are performed using MassLynx, ver. 4.1 and TargetLynx Application Manager. Analytes are quantified against a reference standard by applying the isotope dilution technique. The reference standard for PFAS quantification is prepared with each batch of test samples. The limits of quantification range are $0.01-0.5 \mathrm{ng} / \mathrm{mL}$. Figures 2 and 3 show some examples of chromatograms, which illustrate the separation of perfluorocarboxylic acids and perfluorosulfonate compounds typically found in human serum.

As required by the internal control quality, each laboratory bottle and vial is tested before first use: washing acetonitrile is added, concentrated, and analyzed. If it is PFAS-free, then the glassware can be used. According to
TABLE 1: Water alliance 2695 HPLC pump gradient timetable.

\begin{tabular}{lcc}
\hline Time & A\% & B\% \\
\hline 0.00 & 90 & 10 \\
0.10 & 0 & 100 \\
4.00 & 0 & 100 \\
7.00 & 90 & 10 \\
\hline
\end{tabular}

TABLE 2: MS tune conditions.

\begin{tabular}{lc}
\hline Voltages & \\
$\quad$ Capillary $(\mathrm{KV})$ & 1 \\
\hline Temperatures & \\
$\quad$ Source temp $\left({ }^{\circ} \mathrm{C}\right)$ & 120 \\
Desolvation temp $\left({ }^{\circ} \mathrm{C}\right)$ & 450 \\
\hline Gas flow & \\
Desolvation $(\mathrm{L} / \mathrm{hr})$ & 500 \\
Cone $(\mathrm{L} / \mathrm{hr})$ & 25 \\
\hline
\end{tabular}

TABle 3: Acquisition parameters.

\begin{tabular}{lccccc}
\hline Analyte & Trace & $\begin{array}{c}\text { RT } \\
(\mathrm{min})\end{array}$ & $\begin{array}{c}\text { Dwells } \\
(\mathrm{s})\end{array}$ & $\begin{array}{c}\text { Cone } \\
(\mathrm{V})\end{array}$ & $\begin{array}{c}\text { Collision } \\
(\mathrm{V})\end{array}$ \\
\hline PFBA & $213>169$ & 3.60 & 0.02 & 12 & 9 \\
PFPeA & $263>219$ & 3.76 & 0.02 & 15 & 9 \\
PFHxA & $313>269$ & 3.88 & 0.02 & 15 & 10 \\
PFHpA & $363>319$ & 3.97 & 0.02 & 15 & 9 \\
PFOA & $413>369$ & 4.05 & 0.02 & 15 & 11 \\
PFNA & $463>419$ & 4.13 & 0.02 & 15 & 11 \\
PFDA & $513>469$ & 4.23 & 0.02 & 15 & 13 \\
PFUdA & $563>519$ & 4.31 & 0.02 & 15 & 11 \\
PFDoA & $613>569$ & 4.42 & 0.02 & 20 & 12 \\
PFBS & $299>99$ & 3.84 & 0.02 & 40 & 25 \\
PFHxS & $399>99$ & 4.02 & 0.02 & 50 & 30 \\
PFHpS & $449>99$ & 4.08 & 0.02 & 55 & 35 \\
PFOS & $499>80$ & 4.16 & 0.02 & 55 & 45 \\
\hline PFBA ${ }^{13} \mathrm{C}_{4}$ & $217>172$ & 3.60 & 0.02 & 12 & 9 \\
PFHxA ${ }^{13} \mathrm{C}_{2}$ & $315>270$ & 3.88 & 0.02 & 15 & 10 \\
PFHpA ${ }^{13} \mathrm{C}_{4}$ & $367>322$ & 3.97 & 0.02 & 15 & 9 \\
PFOA ${ }^{13} \mathrm{C}_{4}$ & $417>372$ & 4.05 & 0.02 & 15 & 11 \\
PFNA ${ }^{13} \mathrm{C}_{5}$ & $468>423$ & 4.13 & 0.02 & 15 & 11 \\
PFDA $^{13} \mathrm{C}_{2}$ & $515>470$ & 4.23 & 0.02 & 15 & 13 \\
PFUdA $^{13} \mathrm{C}_{2}$ & $565>520$ & 4.31 & 0.02 & 15 & 11 \\
PFDoA $^{13} \mathrm{C}_{2}$ & $615>570$ & 4.42 & 0.02 & 20 & 12 \\
PFHxS $^{18} \mathrm{O}_{2}$ & $403>103$ & 4.02 & 0.02 & 50 & 30 \\
PFOS $^{13} \mathrm{C}_{4}$ & $503>80$ & 4.16 & 0.02 & 55 & 45 \\
\hline & & & & &
\end{tabular}

the quality system, for each batch of test samples (20 test samples in one batch), at least one procedural blank and one quality control sample (QC) are analyzed. The blank 


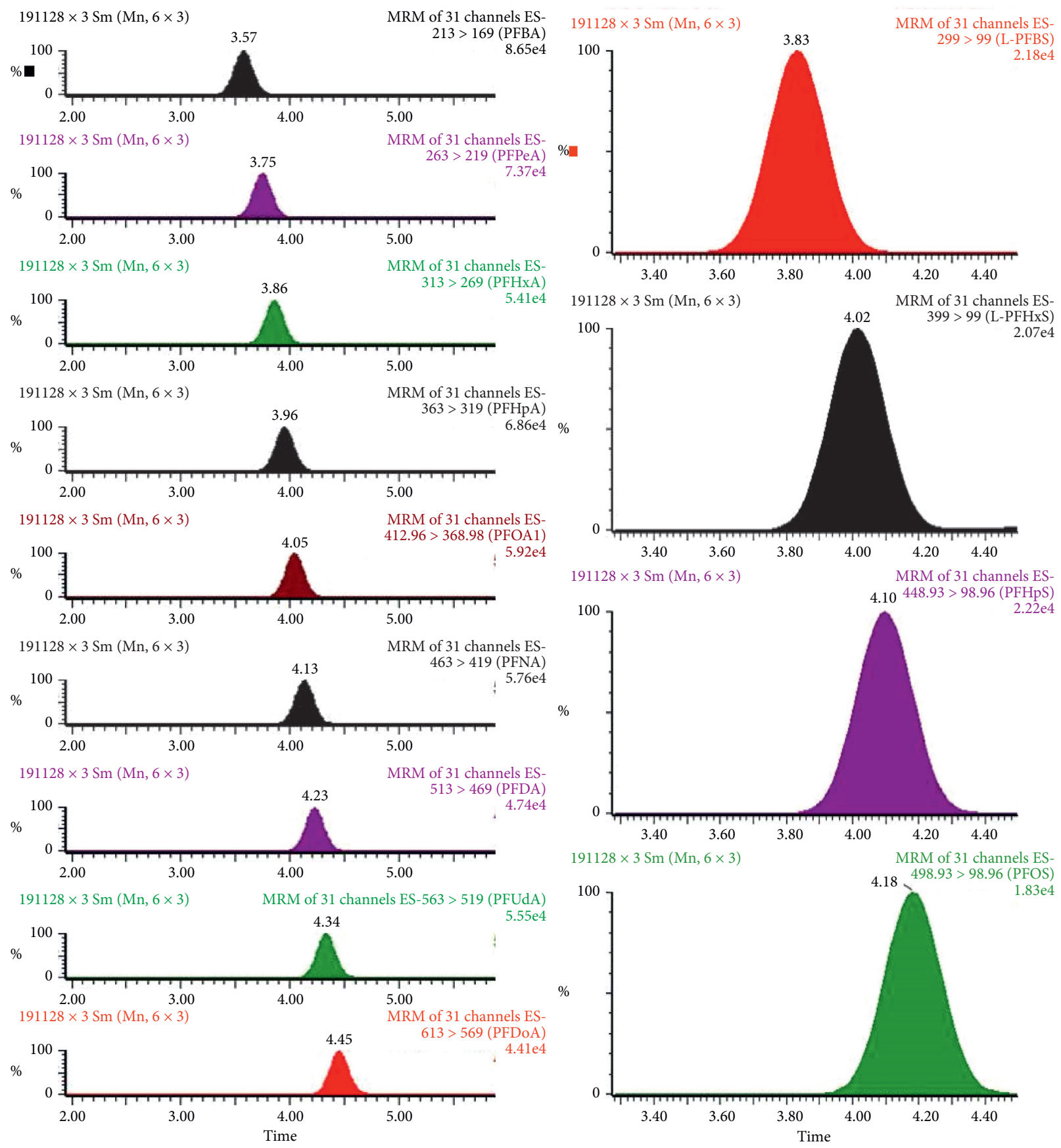

FIgURe 2: Chromatogram (MRM) of PFAS standard solution (perfluorocarboxylic acids (left) and perfluorosulfonate compounds (right)).

and the control sample are spiked, extracted, and treated like the test samples. The concentration of the quality control sample is plotted on a control chart to verify that the procedure is under control. If the analytical batch results to be out of control, results are rejected and causes of the out of control are analyzed and corrected until the system quality control procedures give positive results. We have been using for years the described method for the analysis of PFAS in serum samples within the framework of various national and international projects and in interlaboratory comparison exercises and consider it suitable for routine analysis. Figure 4 shows an example of 

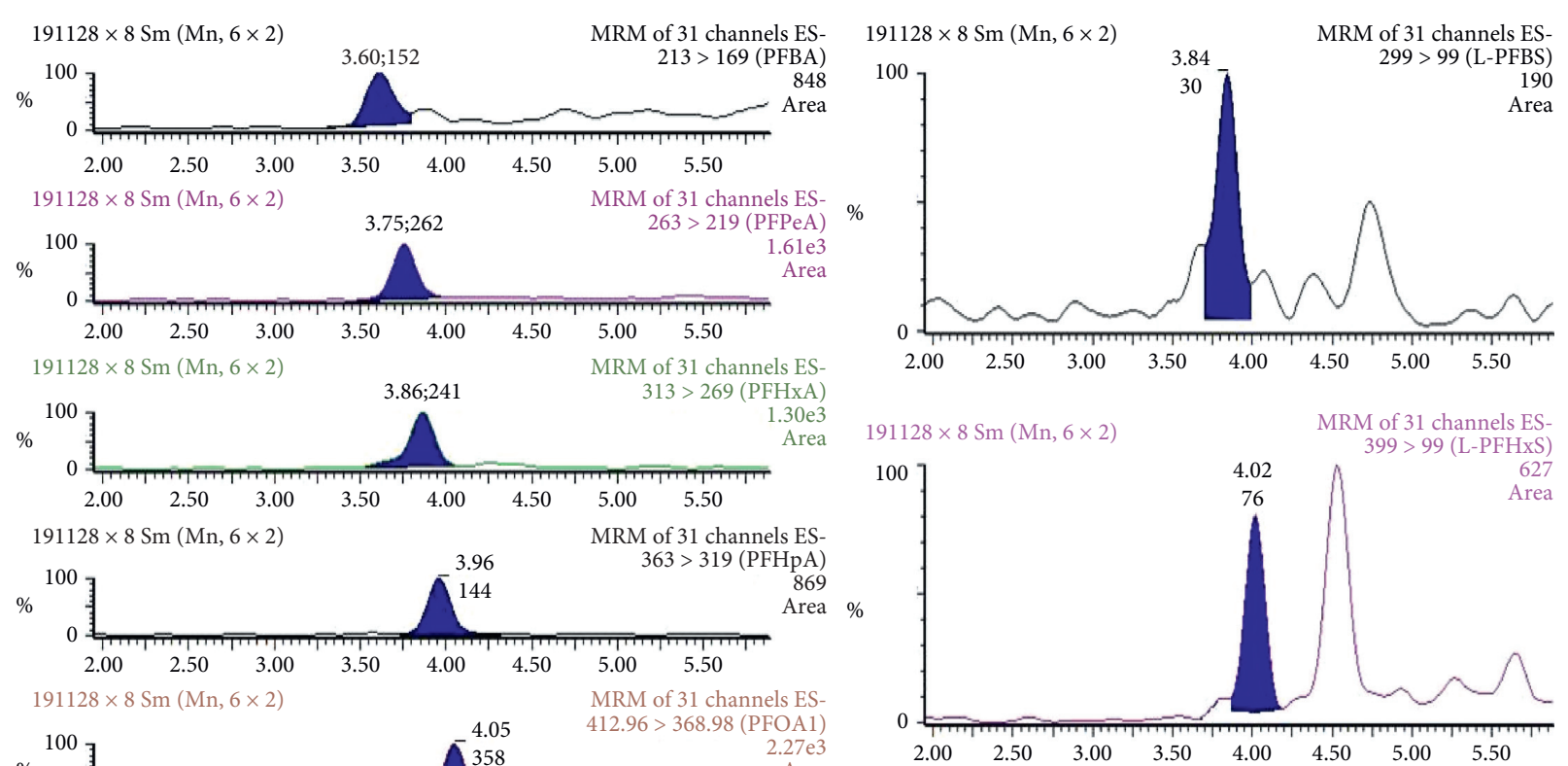

$191128 \times 8 \mathrm{Sm}(\mathrm{Mn}, 6 \times 2)$

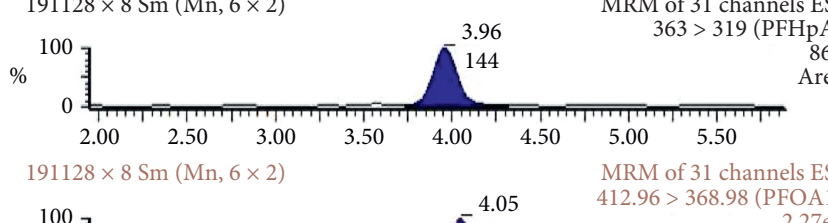

MRM of 31 channels ES
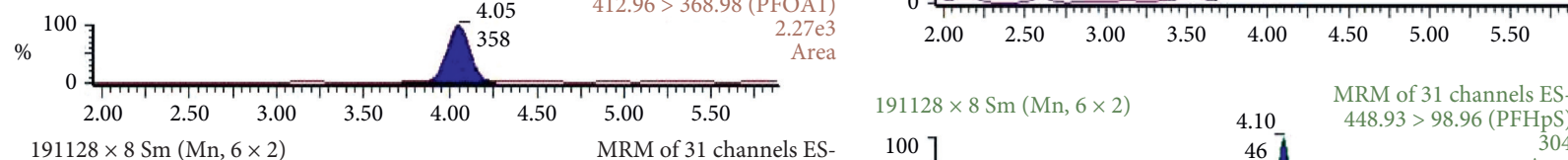

$191128 \times 8 \mathrm{Sm}(\mathrm{Mn}, 6 \times 2) \quad$ MRM of 31 channels ES-
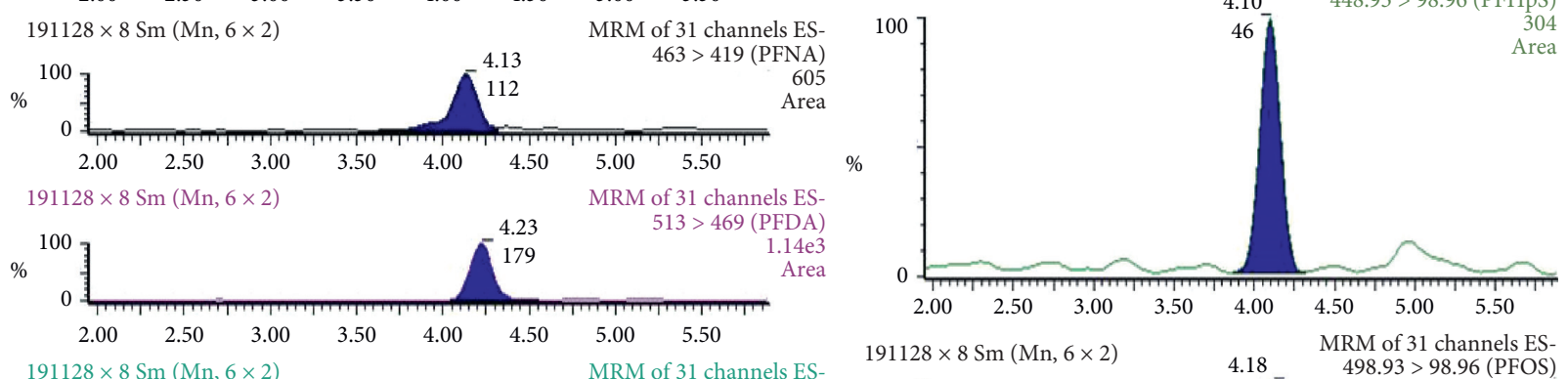

$191128 \times 8 \mathrm{Sm}(\mathrm{Mn}, 6 \times 2) \quad$ MRM of 31 channels ES-
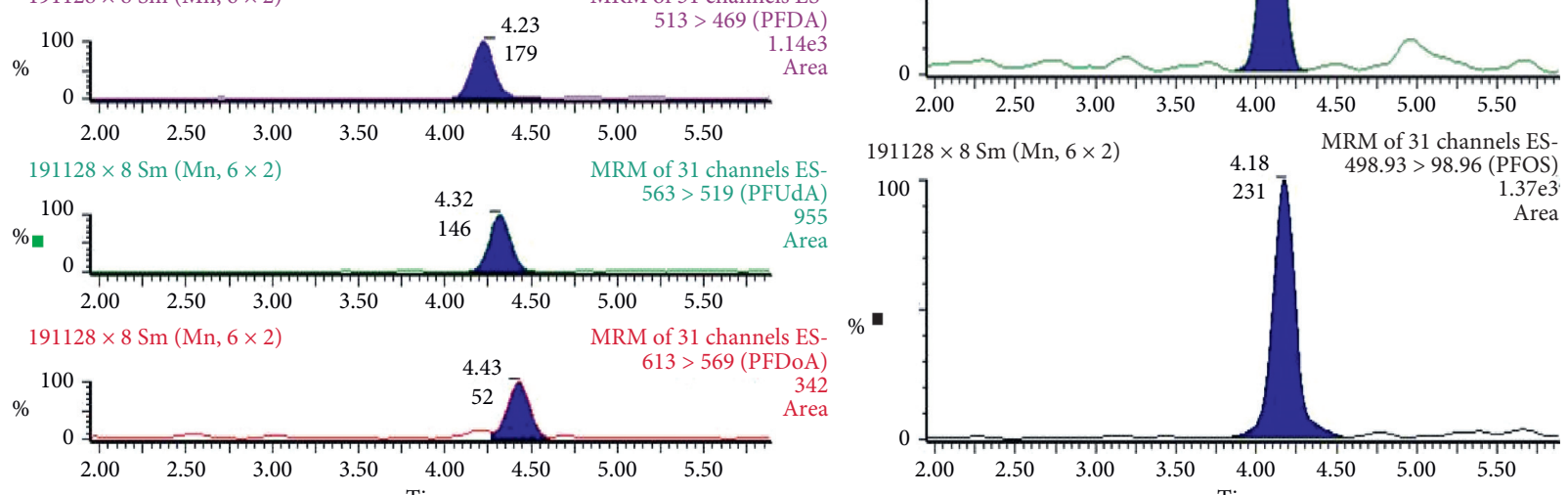

FiguRE 3: Chromatogram (MRM) of a real serum sample (perfluorocarboxylic acids (left) and perfluorosulfonate compounds (right) are present in the sample at very low levels).

the quality control charts we use for each batch of test samples (20 test samples in one batch). We have selected control charts of the most known and most determinable compounds in human serum, PFOS, and PFOA; control charts of all the determined analytes are reported in supplementary data 1 . 


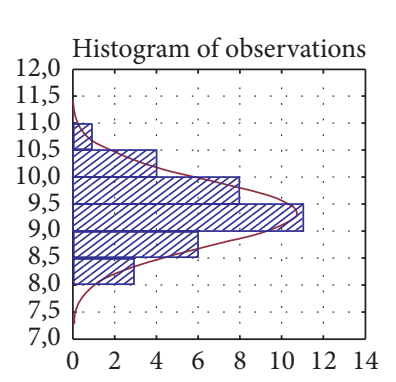

$X$ and moving $R$ chart; variable: PFOS
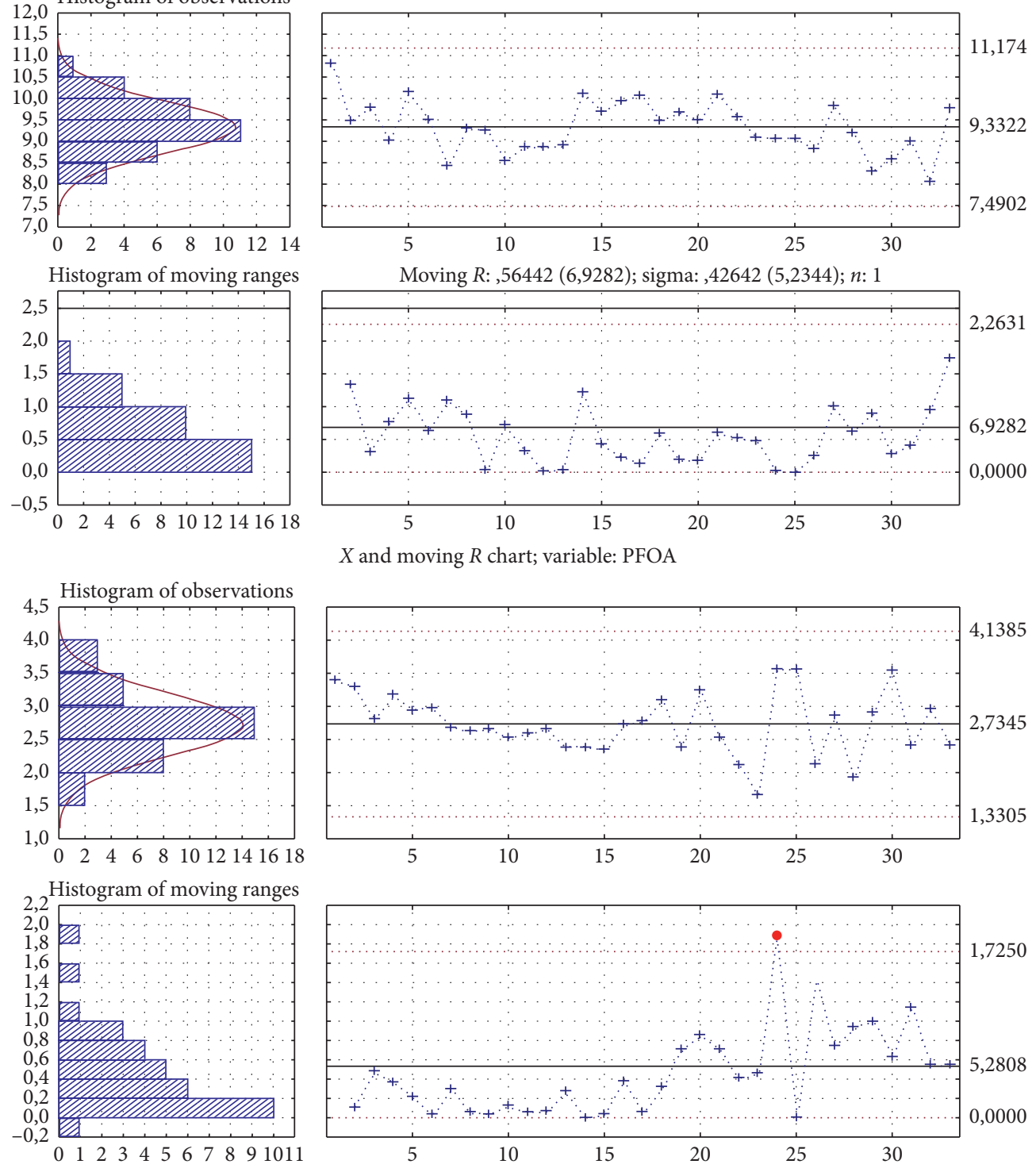

FIgUre 4: Quality control charts of PFOA and PFOS.

\section{Conclusion}

In the present study, a simple and fast analytical method was developed to simultaneously quantify 13 perfluoroalkyl compounds. This method has been developed for the quantification of 13 perfluorocarboxylic acids and perfluorosulfonate compounds (belonging to the class of alkyl per- and polyfluorinated substances (PFAS)) in human serum.

\section{Data Availability}

The reports of the AMAP and HBM4EU (with a good z-score) intercalibration exercises (https://www.hbm4eu.eu; AMAP Ring Exercise Test for Persistent Organic Pollutants in Human Serum https://www.inspq.qc.ca/en/ctq/eqas/ amap/description $[10,15-18])$ support the findings of this study.

\section{Conflicts of Interest}

The authors declare that they have no conflicts of interest.

\section{Acknowledgments}

The authors wish to thank all the participants involved in human biomonitoring national and international studies on PFAS.

\section{Supplementary Materials}

Supplementary data 1: quality control sample charts-quality control charts of PFASs concentrations in the quality control sample were analyzed and collected in our laboratory in the last 5 years. Supplementary data 2: control charts' $z$-score of HBM4EU and AMAP-control charts of $z$-score values obtained in the last 3 years by our laboratory in the interlaboratory 
comparison exercises organized within the HBM4EU project and the Arctic Monitoring and Assessment Programme (AMAP). Supplementary data 3: AMAP report 2016-2017-reports of the interlaboratory comparison exercises organized by the Arctic Monitoring and Assessment Programme (AMAP) in 2016-2017. (Supplementary Materials)

\section{References}

[1] A. B. Lindstrom, M. J. Strynar, and E. L. Libelo, "Polyfluorinated compounds: past, present, and future," Environmental Science \& Technology, vol. 45, pp. 7954-7961, 2011.

[2] E. A. Emmett, H. Zhang, F. S. Shofer et al., "Community exposure to perfluorooctanoate: relationships between serum levels and certain health parameters," Journal of Occupational and Environmental Medicine, vol. 48, pp. 771-779, 2006.

[3] J. Hölzer, O. Midasch, K. Rauchfuss et al., "Biomonitoring of perfluorinated compounds in children and adults exposed to perfluorooctanoate-contaminated drinking water," Environmental Health Perspectives, vol. 116, pp. 651-657, 2008.

[4] K. Hoffman, T. F. Webster, S. M. Bartell, M. G. Weisskopf, T. Fletcher, and V. M. Vieira, "Private drinking water wells as a source of exposure to perfluorooctanoic acid (PFOA) in communities surrounding a fluoropolymer production facility," Environmental Health Perspectives, vol. 119, pp. 92-97, 2011.

[5] K. Steenland, C. Jin, J. MacNeil et al., "Predictors of PFOA levels in a community surrounding a chemical plant," Environmental Health Perspectives, vol. 117, pp. 1083-1088, 2009.

[6] V. Vieira, T. Webster, S. Bartell, K. Steenland, D. Savitz, and T. Fletcher, "PFOA community health studies: exposure via drinking water contaminated by a teflon manufacturing facility," Organohalogen Compound, vol. 70, pp. 730-732, 2008.

[7] ATSDR, "Draft toxicological profile for perfluoroalkyls," 2015, https://www.atsdr.cdc.gov/toxprofiles/tp200.pdf.

[8] Danish Environmental Protection Agency, "Perfluoroalkylated substances: PFOA, PFOS and PFOSA Evaluation of health hazards and proposal of a health based quality criterion for drinking water, soil and ground water. in environmental project no.1665," Chemosphere, vol. 88, pp. 1269-1275, 2015.

[9] R. Barouki, P. D. Gluckman, P. Grandjean, M. Hanson, and J. J. Heindel, "Developmental origins of non-communicable disease: implications for research and public health," Environmental Health, vol. 27, pp. 11-42, 2012.

[10] E. De Felip, A. Abballe, F. L. Albano et al., "Current exposure of Italian women of reproductive age to PFOS and PFOA: a human biomonitoring study," Chemosphere, vol. 137, pp. 1-8, 2015.

[11] J. C. DeWitt, M. M. Peden-Adams, J. M. Keller, and D. R. Germolec, "Immunotoxicity of perfluorinated compounds: recent developments," Toxicologic Pathology, vol. 40, pp. 300-311, 2012.

[12] EFSA, Guidance Document for the Use of the Concise European Food Consumption Database in Exposure Assessment, European Food Safety Authority (EFSA), Parma, Italy, 2008.

[13] S. S. White, S. E. Fenton, and E. P. Hines, "Endocrine disrupting properties of perfluorooctanoic acid," The Journal of Steroid Biochemistry and Molecular Biology, vol. 127, pp. 1626, 2011.

[14] AMAP, "AMAP ring test for persistent organic pollutants in human serum: organized by the institut national de santé publique du québec," Centre de Toxicologie du Québec (Canada) https:// www.inspq.qc.ca/en/ctq/eqas/amap/description, 2001.
[15] A. M. Ingelido, V. Marra, A. Abballe et al., "Perfluorooctanesulfonate and perfluorooctanoic acid exposures of the Italian general population," Chemosphere, vol. 80, pp. 1125-1130, 2010.

[16] A. M. Ingelido, A. Abballe, S. Gemma et al., "Biomonitoring of perfluorinated compounds in adults exposed to contaminated drinking water in the Veneto region, Italy," Environment International, vol. 110, pp. 149-159, 2018.

[17] A. M. Ingelido, A. Abballe, S. Gemma et al., "Serum concentrations of perfluorinated alkyl substances in farmers living in areas affected by water contamination in the Veneto region (Northern Italy)," Environment International, vol. 136, Article ID 105435, 2020.

[18] M. G. Porpora, R. Lucchini, A. Abballe et al., "Placental transfer of persistent organic Pollutants: a preliminary study on mother-newborn pairs," International Journal of Environmental Research and Public Health, vol. 10, pp. 699-711, 2013.

[19] HBM4EU, "Science and policy for a healthy future," https:// www.hbm4eu.eu. 